Pathologe 2008 · [Suppl 2] 29:270-272

DOI 10.1007/s00292-008-1033-z

Online publiziert: 20. August 2008

(c) Springer Medizin Verlag 2008

\section{G. Mikuz}

Institut für Pathologie der Medizinischen Universität Innsbruck, Österreich

\section{Hodentumoren}

\section{Prognostische Faktoren}

In den Ländern Westeuropas ist die Inzidenz der Hodentumoren in den letzten Jahren dramatisch gestiegen. Im Gegensatz dazu ist das Interesse der Pathologen für diese biologisch faszinierenden Geschwülste stark gesunken. Eine mögliche Erklärung für dieses Desinteresse ist nicht nur die Seltenheit dieser Tumoren, sondern auch die unbegründete Ansicht, die Pathologie sei für die therapeutischen Entscheidungen irrelevant.

Keimzelltumoren, die auf den Hoden beschränkt sind und noch nicht metastasiert haben (klinisches Stadium I) ${ }^{1}$, können entweder sofort (primär) adjuvant behandelt werden, oder aber die Behandlung wird erst beim Auftreten von Metastasen (klinisch allgemein als „Rezidiv“ bezeichnet) eingesetzt. Beide Strategien gehören zum Standardmanagement und erreichen Heilungsraten von 98-99\% $[4,6]$.

In den USA wird vornehmlich die retroperitoneale Lymphknotendissektion durchgeführt. Dank des leichteren $\mathrm{Zu}$ gangs zu den Gesundheitseinrichtungen

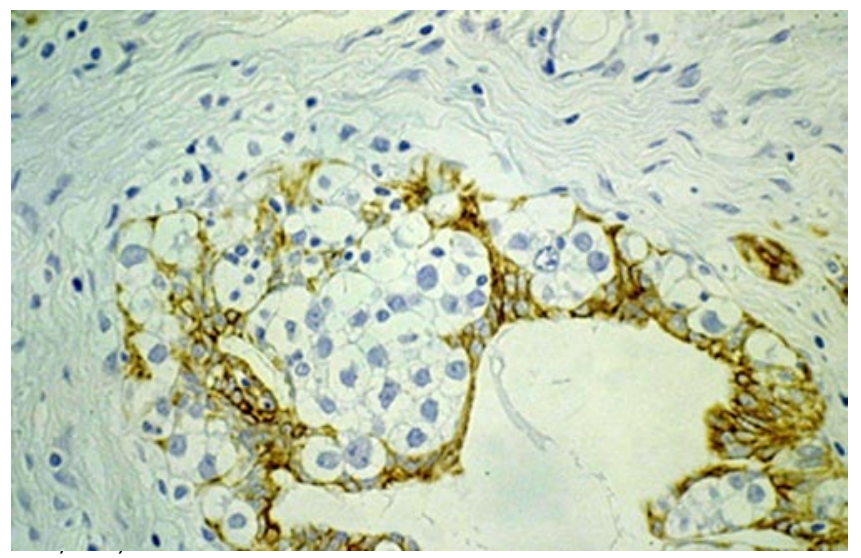

Abb. $1<$ Invasion des Rete testis durch Seminomzellen. Die Reteepithelien sind mit Zytokeratinantikörpern bräunlich gefärbt (Immunhistochemie, Vergr. 400:1)
Die 2 wesentlichen Risikofaktoren des Seminoms und dessen morphologischen Varianten (das spermatozytäre Seminom selbstverständlich ausgenommen) sind ([15] A Abb. 1):

- der Tumordurchmesser $\leq 4 \mathrm{~cm}$ und

- die Infiltration des Rete testis.

Ist nur einer dieser Faktoren vorhanden, ist die Häufigkeit des Rezidivs nur um wenige Prozentpunkte höher als bei Tumoren ohne diese Merkmale. Sind hingegen beide Faktoren vorhanden, steigt die Rezidivrate von $15 \%$ auf $32 \%$ [15]. Überhaupt zeigen alle statistischen Untersuchungen, dass die Infiltration des Rete testis den Verlauf der Krankheit am stärksten negativ beeinflusst (- Tab. 1). Selbst nach adjuvanter Carboplatin-Behandlung haben Patienten mit diesem Risikofaktor schlechtere Chancen, rezidivfrei zu bleiben, als jene ohne Risikofaktoren [2]. Die Gefäßinvasion ( $\mathrm{pT}$ 2) ist im Gegensatz zu den anderen Keimzelltumoren beim Seminom kein wichtiger prognostischer Faktor $[3,6,9]$.

Die Bedeutung des Alters für die Prognose ist umstritten: In einigen Untersuchungen wird das Alter unter 30 Jahre als Risikofaktor betrachtet $[3,15]$, andere können sich dieser Meinung nicht anschließen [14]. Viele Diskrepanzen in den diesbezüglichen Untersuchungen beruhen auf den Unterschieden in der Zahl der Patienten und in der Länge der Beobachtungszeit.

Die in den länger zurückliegenden Untersuchungen gezeigte Korrelation zwischen dem günstigen Krankheitsverlauf bei starker und ungünstigem Verlauf bei schwacher oder fehlender lymphozytärer Infiltration des Tumors [10] konnte in 
späteren Arbeiten [12] nicht mehr bestätigt werden. Ebenfalls fragliche bzw. prognostisch unwichtige Faktoren sind die synzytiotrophoblastären Riesenzellen und das „anaplastische Seminom“, das aus der WHO-Klassifikation gestrichen wurde [5], aber dennoch weiterhin in den pathologischen Diagnosen zu finden ist.

Bei den nichtseminomatösen Keimzelltumoren ist die Ausgangslage etwas dramatischer: $50 \%$ der Patienten haben zum Zeitpunkt der Diagnose bereits retroperitioneale Lymphknotenmetastasen. Die Lymphknotendissektion und/oder die adjuvante Chemotherapie sind die bevorzugten Behandlungsmethoden. Dennoch haben englische Kliniker bereits vor Jahren gezeigt, dass eine erst beim Rezidiv eingesetzte Chemotherapie gleichgute Ergebnisse zeigt wie die adjuvante Chemotherapie, sodass auch bei dieser Tumorgruppe die Beobachtungsstrategie ohne Schaden für den Patienten angewendet werden kann [4]. Knapp die Hälfte (48\%) der Patienten unter Beobachtung und $1 / 4$ der adjuvant behandelten wird allerdings Metastasen entwickeln.

Von der „European Germ Cell Cancer Consensus Group“ wird bei nichtseminomatösen Keimzelltumoren nur die Gefäßinvasion als negativer prognostischer Faktor anerkannt [9]. Dennoch zeigen viele Studien [4, 7, 8], dass der Anteil des embryonalen Karzinoms ebenfalls wichtig ist: Übersteigt der Anteil 70\%, ist auch ohne erkennbare Gefäßinvasion bei $80 \%$ der Betroffenen mit Metastasen zu rechnen. Die Anwesenheit von Dottersacktumor- $[7,13]$ und Teratomanteilen $[1]$ in einem Keimzellmischtumor hat hingegen eine günstige prognostische Auswirkung, die allerdings nicht so ausgeprägt ist, wie die negative Wirkung der oben genannten Faktoren (• Tab. 2).

Aufgrund dieser Eigenschaften kann sogar ein Regressionskoeffizient nach folgender Formel errechnet werden:

Index $=1,5 \times$ Veneneinbruch $+0,9$ Lymphgefäßeinbruch $-0,8$ Dottersacktumor $+1,4 \times$ embryonales Karzinom

wobei die Anwesenheit und Abwesenheit eines Merkmals jeweils mit 1 oder o bewertet werden [4].

Demnach muss man bei nichtseminomatösen Keimzelltumoren gründlich

Pathologe 2008 • [Suppl 2] 29:270-272 DOI 10.1007/s00292-008-1033-z

(c) Springer Medizin Verlag 2008

\section{G. Mikuz \\ Hodentumoren. Prognostische Faktoren}

\section{Zusammenfassung}

Keimzelltumoren, die auf den Hoden beschränkt sind, können entweder sofort nach Orchiektomie adjuvant behandelt werden, oder aber die Behandlung wird erst beim Auftreten von Metastasen eingesetzt (,,Surveillance-Strategie"). Beide Strategien gehören zum Standardmanagement und erreichen Heilungsraten von 98-99\%. Der Vorteil der Surveillance-Strategie ist es, dass nur jene Betroffenen behandelt werden, die eine Behandlung wirklich benötigen. Gegen die generelle Anwendung der toxischen Chemotherapie spricht vor allem die Tatsache, dass nur die Hälfte der Hodenkrebspatienten diese Therapie auch wirklich benötigt. Für die Beobachtungsstrategie besonders geeignet sind Patienten, deren Tumoren keine morphologischen Risikofaktoren zeigen. Beim klassischen Seminom ist der wichtigste Prädiktor des Rezidivs die Invasion des Rete testis, gefolgt vom Tumordurchmesser $(\geq 4 \mathrm{~cm})$.

\section{Testicular tumors. Predictive factors}

\section{Abstract}

Patients with germ cell tumors of the testis can be given adjuvant treatment immediately after orchidectomy or put under surveillance with definitive treatment deferred until relapse. Both therapies are equally successful (success rate 98-99\%), with surveillance alone, however, only approximately $50 \%$ of cases need toxic chemotherapy. The surveillance strategy is especially successful in patients with tumors which do not have morphological predictors of metastases. In seminomas the strongest predictor of metastases is tumor invasion of the rete testis followed by the size $(\varnothing \geq 4 \mathrm{~cm})$ of the tumor. Vascular invasion, the most important predictor in non-seminomatous germ cell tumors, is less
Die Gefäßinvasion hat beim Seminom nur eine geringe prädiktive Aussagekraft, bei nichtseminomatösen Keimzelltumoren hingegen ist sie der sicherste Metastasenprädiktor, gefolgt von der Anwesenheit und der Menge des embryonalen Karzinoms. Teratom- und Dottersackanteile in den Mischtumoren werden als günstige Faktoren angesehen. Die statistische Aussagekraft ist aber weit schwächer als die der erstgenannten. Bei den seltenen Tumoren des Gonadenstromas verwendet man morphologische Merkmale nur für die manchmal schwierige Bestimmung der Dignität. Sicher maligne sind Leydig- und Sertoli-Zell-Tumoren nur, wenn ihr Durchmesser $\geq 5 \mathrm{~cm}$ beträgt.

Schlüsselwörter

Keimzelltumoren · Surveillance $\cdot$ Rete-testisGefäßinvasion · Rezidiv important in seminomas. The second most important predictor in mixed tumors is the presence or absence and the amount of embryonal carcinoma. The presence of teratomas and yolk sac tumors are considered to be predictors of a favorable course of the disease. The statistical impact of these markers is, however, not very strong. The only reliable predictor of malignancy of the gonadal stromal tumors is the size and tumors with a diameter $\geq 5 \mathrm{~cm}$ are always malignant.

\section{Keywords}

Testicular tumors · Surveillance $\cdot$ Rete testis invasion · Vascular invasion · Relapse 
Tab. 1 Prognostisch ungünstige Faktoren des Seminoms und deren statistische Wertigkeit. (Mod. nach [3])

\begin{tabular}{|ll}
\hline Eigenschaft & p \\
Rete-testis-Invasion & 0,03 \\
\hline Alter $\leq 30$ Jahre & 0,21 \\
\hline Tumordurchmesser $\geq 4 \mathrm{~cm}$ & 0,29 \\
\hline Gefäßinvasion & 0,67 \\
\hline p:Wahrscheinlichkeit. & \\
\hline
\end{tabular}

Tab. 2 Prognostisch ungünstige und günstige (kursiv) Faktoren von nichtseminomatösen Keimzelltumoren und deren statistische Wertigkeit. (Mod. nach [4])

Eigenschaft

Blutgefäßeinbruch p Lymphgefäßeinbruch $<0,001$ Dottersacktumor $<0,001$ Embryonales Karzinom 0,007 p:Wahrscheinlichkeit.

\section{Infobox 1}

\section{Morphologische Malignitätsmerkmale von Leydig- und Sertoli-Zell-Tumoren \\ - Durchmesser $\geq 5 \mathrm{~cm}$ \\ - Hohe Mitoserate \\ - Gefäßeinbrüche \\ - Infiltration der Umgebung \\ - Nekrose}

Nur der Durchmesser ist als Einzelkriterium absolut zuverlässig; von den anderen Merkmalen müssen zumindest 2 vorhanden sein.

nach Gefäßeinbrüchen suchen und den prozentualen Anteil des embryonalen Karzinoms grob schätzen. Die Abschätzung des Metastasenrisikos (hoch/niedrig) im Endbefund ist für die Wahl der Therapie sehr hilfreich.

Für die Therapie besonders wichtig ist das Erkennen somatischer Neoplasien, die sich gelegentlich in einem Teratom entwickeln (WHO: „teratoma with somatic typ malignancies“; [5]). Die somatische Differenzierung wird klinisch relevant, wenn bei schwacher Vergrößerung (Objektivvergr. 4:1) mehr als die Hälfte eines Gesichtsfeldes von derartigen Tumorformationen eingenommen wird [10]. Am häufigsten werden Neuroblastome, primitive neuroektodermale Tumoren, Nephroblastome und Rhabdomyo- oder andere Sarkome beobachtet [10]. Derartige Tumoren verlangen nach einer maßgeschneiderten
Therapie und dürfen weder nach dem üblichen Chemotherapieprotokoll für Keimzelltumoren behandelt noch unter Beobachtung gestellt werden [11].

Tumoren des Gonadenstromas sind eher selten. Aus diesem Grund fehlen sichere Kriterien für die Unterscheidung zwischen gutartigen und bösartigen Leydig- bzw. Sertoli-Zell-Tumoren. Das einzige sichere Zeichen der Malignität sind ein Durchmesser $>5 \mathrm{~cm}$ und Metastasen. Die hohe mitotische Aktivität, die Gefäßinvasion, die Nekrose und die Infiltration der Umgebung sind nur dann aussagekräftig, wenn zumindest 2 dieser Eigenschaften zusammentreffen ( $\bullet$ Infobox 1; [10]).

\section{Korrespondenzadresse}

\section{Prof. Dr. G. Mikuz}

Institut für Pathologie der Medizinischen Universität Innsbruck

Müllerstr. 44, 6020 Innsbruck, Österreich

Gregor.Mikuz@i-med.ac.at

Interessenkonflikt. Der korrespondierende Autor gibt an, dass kein Interessenkonflikt besteht.

\section{Literatur}

1. Alexandre J, Fizazi K, Mahé C et al. (2001) Stage I non-seminomatous germ-cell tumours of the testis: identification of a subgroup of patients with a very low risk of relapse. Eur J Cancer 37: 576-582

2. Aparicio J, Germà JR, García del Muro X et al. (2005) Risk-adapted management for patients with clinical stage I seminoma: the Second Spanish Germ Cell Cancer Cooperative Group study. J Clin Oncol 23: 8717-8723

3. Choo R, Thomas G, Woo T et al. (2005) Long-term outcome of postorchiectomy surveillance for Stage I testicular seminoma. Int J Radiat Oncol Biol Phys 61: 736-740

4. Cullen M (1996) Management of stage I non seminoma: surveillance and chemotherapy. in Horwich A (ed) Testicular cancer: investigation and management, 2nd edn. Chapman \& Hall, London, pp 149-166

5. Eble JN, Sauter G, Epstein JI, Sesterhenn IA (eds) (2007) WHO Classification of tumors. Pathology \& genetics. Tumors of the urinary system and male genital organs. IARC, Lyon, pp 218-219

6. de Witt R, Fizazi K (2006) Controversies in the management of clinical stage I testis cancer. J Clin Oncol 24: 5482-5492

7. Freedman LS, Parkinson MC, Jones WG et al. (1987) Histopathology in the prediction of relapse of patients with stage I testicular teratoma treated by orchidectomy alone. Lancet 2: 294-298

8. Heidenreich A, Sesterhenn IA, Mostofi FK et al. (1999) Histopathologische und biologische Prognosefaktoren nichtseminomatöser Keimzelltumoren im klinischen Stadium I. Urologe [A] 38: 168-178
9. Krege S, Beyer J, Souchon R et al. (2008) European consensus conference on diagnosis and treatment of germ cell cancer: a report of the second meeting of the European Germ Cell Cancer Consensus group (EGCCCG): part I. Eur Urol 153: 478-496

10. Mikuz G, Colecchia M (2007) Tumors of the testis and paratesticular structures. In Mikuz G (ed) Clinical pathology of urological tumors. Informa, London, pp 161-214

11. Motzer RJ, Amsterdam A, Prieto V et al. (1998) Teratoma with malignant transformation: diverse malignant histologies arising in men with germ cell tumors. J Urol 159: 133-138

12. Parker C, Milosevic M, Panzarella T et al. (2002) The prognostic significance of the tumour infiltrating lymphocyte count in stage I testicular seminoma managed by surveillance. Eur J Cancer 38: 20142019

13. Porcaro AB, Zecchini Antoniolli S, Novella G et al. (2001) Opzioni terapeutiche per i tumori germinali non seminomatosi del testicolo (TGNST) in stadio clinico I. Uno studio retrospettivo su 75 pazienti. Arch Ital Urol Androl 73: 177-180

14. Valdevenito JP, Gallegos I, Fernández C et al. (2007) Correlation between primary tumor pathologic features and presence of clinical metastasis at diagnosis of testicular seminoma. Urology 70: 777780

15. Warde P, Specht L, Horwich A et al. (2002) Prognostic factors for relapse in stage I seminoma managed by surveillance: a pooled analysis. J Clin Oncol 20: 4448-4452 